\title{
ILP-Based Board-Level Routing of Multi-Terminal Nets for Prototyping Reconfigurable Interconnect
}

\author{
A. Kirschbaum, J. Becker, M. Glesner \\ Institute of Microelectronic Systems \\ Darmstadt University of Technologie \\ \{kirschbaumlbeckerlglesner\} @mes.tu-darmstadt.de
}

\begin{abstract}
For the board-level routing of intermodule connections with reprogrammable devices in a prototyping environment we present an Integer Linear Programming (ILP) model. It is solvable in polynomial time for architectures consisting of two routing devices even for multi-terminal nets. A net decomposition strategy is presented to handle all remaining infeasible problems. In contrast to previous work this approach allocates a minimum of additional port resources and thus significantly improves the routability of multi-terminal net dominated designs.
\end{abstract}

Keywords: Reconfigurable Interconnect, Rapid-Prototyping, Board-Level Routing, ILPModel

\section{INTRODUCTION}

Advances in integrated circuit technology resulted in large and fast reconfigurable devices such as field-programmable gate arrays (FPGAs) and fieldprogrammable interconnection components (FPICs) and have enabled designers to create a new class of open-architecture reconfigurable hardware platforms. Whereas FPGAs are mainly used for prototyping digital logic designs, FPICs allow the arbitrary interconnection of almost any kind of digital system modules.

A typical prototyping system consists of a set of communicating processing elements such as processors, custom logic modules in multiple FPGAs, memory devices, and I/O circuits. In order to preserve flexibility, the system topology is often personalized with a set of FPICs. The interconnection of the system 
modules and consequently the configuration of the FPICs is hereinafter referred to as board-level routing problem.

Chan and Schlag examined the related problem of net routing in an FPGAbased computing system for the special case of prototyping platforms which are more restrictive with respect to the number of communication links than the approach presented in the following Chan and Schlag, 1993.

Mak and Wong Mak and Wong, 1995b investigated the board-level routing problem for FPGA-based logic emulation systems Varghese et al., 1993. For the case where all nets are two-terminal nets, they presented an $O\left(n^{2}\right)$-time optimal algorithm ( $n=$ number of intermodule nets) for configuring the FPICs. This is in contrast to previous approaches which are based on greedy heuristics Slimane-Kadi et al., 1994. The algorithm, which basically finds an Euler circuit in a multigraph, guarantees $100 \%$ routing completion if the number of intermodule nets does not exceed the I/O-capacity of the FPGAs. Furthermore, they proved that the BLRP with multi-terminal nets in the presence of $k$ routing devices $(k>2)$ is $N P$-complete. In Mak and Wong, 1995a the routing of $m$ terminal nets is relaxed by decomposing them into $m-1$ two-terminal nets. A network flow-based algorithm is used to determine a decomposition whenever a feasible one exists. The routing of the resulting two-terminal nets is then completed with the optimal algorithm of Mak and Wong, 1995b. Obviously, this approach suffers from the extensive allocation of additional port resources at the FPGAs and FPICs. Unfortunately, this property becomes crucial for the feasibility of the BLRP in presence of many multi-terminal nets.

In this paper we will investigate the BLRP for system architectures with coarse grained modules which occur for example in embedded systems with bus-dominated interconnection topologies Yen and Wolf, 1995 Ortega and Borriello, 1997 Gasteier and M., 1996. The coarse granularity as opposed to that found in FPGA-based logic emulation systems allows us to reduce the number of independent switching devices in our prototyping environment to two. It can be shown that the BLRP can be solved in polynomial time for this instance of the problem even in the presence of multi-terminal nets. We will present an Integer Linear Programming (ILP) model Lawler, 1976 for determining a feasible router configuration whenever one exists without splitting any net. For cases where the problem turns out to be infeasible due to resource conflicts we propose an iterative net decomposition strategy. This two phases approach consists of a heuristic to find a suitable net for splitting and a computationally efficient ILP formulation for determining the module at which the net is to be split as well as a valid router configuration. Again, this approach allocates only as much resources as necessary for solving the BLRP which will significantly increase routability for a particular prototyping environment compared to the approach from Mak and Wong, 1995a. 
In Section 2. we define a generic system model as basis for the BLRP and introduce our prototyping environment. The formal definition of the BLRP is given in Section 3.. The ILP-based algorithm for routing multi-terminal nets without splitting is presented in Section 4. A detailed discussion of the net decomposition strategy which is applied if the primary BLRP turns out to be infeasible follows in Section 5. . Some experimental results and remarks about the complexity of the presented algorithms are given in Section 6.. Section 7. concludes the paper.

\section{A GENERIC PROTOTYPING ENVIRONMENT}

The board-level routing problem discussed in this paper is not specific for a particular prototyping environment but also occurs in many switch-based system architectures. Therefore we introduce a generic architecture of a reconfigurable system as basis for the board-level routing problem.

Such an execution unit $E U(M, P, K)$ consists of a set of functional modules $M$, a set of ports $P$, and a set of routers $K$ (Figure 1.1 ). The architectures completely described by the parameters $M, P, K$ from which the following can be derived: Each module $m \in M$ contains $B=P / M$ ports which are used for intermodule communication. They are partitioned into $|K|$ equally sized disjoint sets $P_{m}^{1} \ldots P_{m}^{|K|}$ which denotes the $i$ th portset at module $m$. Let $P_{m}=\bigcup_{i=1}^{|K|} P_{m}^{i}$ the set of all ports of module $m$ and $P^{i}=\bigcup_{m=1}^{|M|} P_{m}^{i}$ the set of all ports with a physical connection to router $i$.

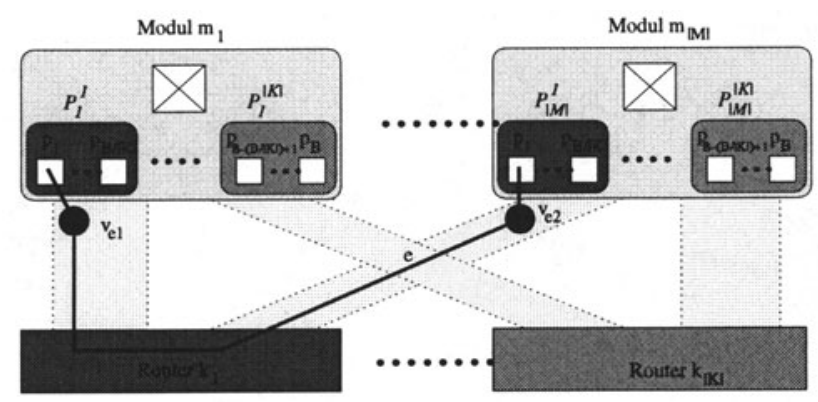

Figure 1. 1 Generic Architecture of an Execution Unit EU(MR).

Intermodule connections can be established between any two ports $p_{x}, p_{y} \in$ $P^{i}$ via the non-blocking switching matrix of router $i$. As there is no physical interconnection between the routers, it is impossible to connect any ports $p_{x}, p_{y}$ with $p_{x} \in P^{i}, p_{y} \in P^{j}$ and $i \neq j$. Additionally, any two ports $p_{x}, p_{y} \in P_{m}$ can also be connected via non-blocking intramodule switching resources (e.g. router, FPGA). 
A rapid prototyping system which complies with the generic architecture of an execution unit is REPLICA ${ }^{1}$ Kirschbaum and Glesner, 1997, a Rapid Prototyping System focusing on inter-/processor-module communication emulation. The system is integrated in a design environment for embedded mixed hardware/software systems (DICE Gasteier et al., 1996). Due to the coarse granularity of the target system modules (processors, memories, ASICs etc.) which are to be connected and the large number of available $\mathrm{I} / \mathrm{O}$ ports of the switching devices (320 ports icu, 1996), the prototyping system consists of only two independent routers. Thus REPLICA can be modeled as an execution unit $E U(6,636,2)$. This prototyping environment facilitates design space exploration and validation of communication links. The reprogrammable system architecture allows prototyping of different topologies, communication types and protocols and is supported by a powerful toolset for automatic system configuration. A reconfigurable hardware monitor Kirschbaum et al., 1998 is available for non-intrusive observation and extraction of real-time data about I/O-channel activities.

\section{PROBLEM DESCRIPTION}

To prototype a system, it has to be mapped to the execution unit. However, the interconnection of the system modules can be established by different switching devices, i.e. appropriate routers have to be selected for the communication links of the system. Thus, an important task is to find a mapping of the target system topology to the reconfigurable interconnection architecture of the $E U$ which avoids router congestions. In the following section we give some basic definitions of the underlying system model, and a formal description of the above-mentioned board-level routing problem.

The topology of the target system, which is to be mapped onto an execution unit $E U$, can be described with an undirected graph $N G(V, E)$ termed netgraph. The nodes $V$ of this graph represent a set of terminals which are connected via hyperedges $E$ termed nets. A net $e$ consists of a set of terminals $V_{e} \subset V$. It represents a physical connection of two or more different modules of a set $M_{e} \subset M$ of an execution unit $E U(M, P, K)$. Each terminal is unambiguously mapped to a module by $\psi: V \rightarrow M$ which is determined by the target system architecture. Thus, our task is to assigns each terminal of a net to a port of a module. We can formulate the BLRP as follows:

Definition Feasible Board-Level Routing Problem: Given an execution unit $E U(M, P, K)$ and a netgraph $N G(V, E)$ : Does there exist an assignment $\phi: V \rightarrow P$ of terminals to ports, i.e. a mapping of the netgraph $N G$ onto the execution unit $E U$, such that the following constraints are not violated: 
1. Unambiguousness: Each port $p$ of a module $m$ can only be allocated to at most one terminal $v$ :

$$
\forall v_{x}, v_{y} \in V: \phi\left(v_{x}\right) \neq \phi\left(v_{y}\right) .
$$

2. Connectivity: The connectivity must be preserved:

$$
\forall e \in E \forall v \in e: \phi(v) \in P_{\psi(v)} .
$$

That means that a terminal which maps onto a module $m$ obviously has to be connected to a port from $P_{m}$.

Also because there is no physical connection between the routers $K$, all terminals $v$ of a net $e$ must be connected via the same router:

$$
\begin{array}{r}
\forall e \in E \forall v_{x}, v_{y} \in e \exists i \in\{1, \ldots,|K|\}: \\
\phi\left(v_{x}\right) \in P^{i} \wedge \phi\left(v_{y}\right) \in P^{i}
\end{array}
$$

Obviously, there are several equivalent solutions for the same board-level routing problem (if a solution exists at all), because the connectivity constraint forces only the allocation of a portset but not of a specific port within that set. Moreover, there are no costs associated with a particular mapping, because all routing resources within the generic architecture are equivalent. The described problem is a pure feasibility problem and thus there exists no cost function.

\section{BOARD-LEVEL ROUTING}

Board-level routing in our prototyping environment is relaxed with respect to the general case, because of the architecture of REPLICA. Since the system consists of only two routers $(K=2)$, the terminals have to be assigned to only two different sets of ports.

\subsection{ILP FORMULATION}

Given a netgraph $N G(V, E)$ and an execution unit $E U(M, P, 2)$, we present a computationally efficient ILP formulation for solving the board-level routing problem.

The mapping $\psi$ of the terminals to the two different sets of ports can easily be modeled with a set of binary decision variables $X=\left\{x_{i} \mid v_{i} \in V\right\}$. It is $x_{i}=0$, if terminal $v_{i}$ is assigned to a port $p \in P^{1}$ and $x_{i}=1$, if $p \in P^{2}$. In order to guarantee the semantic correctness of the mapping the following constraints have to be fulfilled:

- For preserving the connectivity of the target system we require 


$$
\forall e \in E \forall v_{i}, v_{j} \in e: x_{i}=x_{j} .
$$

That means all terminals $v_{i}$ of a net $e$ are connected via the same router.

- The number of terminals assigned to port sets $P_{m}^{1}, P_{m}^{2}$ must not exceed their cardinality. Thus, the resource constraints are

- router 1:

$$
\forall m \in M: \sum_{i: \psi\left(v_{i}\right)=m}\left(1-x_{i}\right) \leq\left|P_{m}^{1}\right|=\frac{|P|}{2}
$$

- router 2:

$$
\forall m \in M: \sum_{i: \psi\left(v_{i}\right)=m} x_{i} \leq\left|P_{m}^{2}\right|=\frac{|P|}{2} .
$$

Each assignment $\phi: V \rightarrow P$ which does not violate these constraints is termed feasible.

\section{SPLITTING OF MULTI-TERMINAL NETS}

It turns out that the ILP problem, as defined in the previous section, may be infeasible due to router congestions. This means that the original netgraph $N G(V, E)$ cannot be mapped to the execution unit due to resource conflicts.

The netgraph shown in Figure 1.2 consisting of one 4-terminal net, one 3-terminal net, and one 2-terminal net, for example, leads to an infeasible ILP problem. This is non-obvious: although the overall number of terminals per module is not exceeded, there are router resource problems due to the fact that all terminals of a net must be connected via the same router.

Splitting of multi-terminal nets is a technique to enable the mapping of netgraphs even if the ILP-problem turned out to be infeasible. The specific property of intramodule connectivity of the execution unit is used for that purpose: All ports $p$ of a module $m\left(p \in P_{m}\right)$ can be internally connected to each other via a non-blocking switching matrix without allocating resources outside of the module. When splitting a net, a terminal $v_{x}$ is duplicated with a terminal $v_{x^{\prime}}$ at the splitting module $m_{s}$. The associated ports $p_{x}=\phi\left(v_{x}\right)$ and $p_{x^{\prime}}=\phi\left(v_{x^{\prime}}\right)$, respectively, are connected via the internal routing resources such that $p_{x} \in P^{i}$ and $p_{x^{\prime}} \in P^{j}$ with $i \neq j$. Hence, net $e$ may be accessed from router $i$ and $j(i, j \in\{1, \ldots,|K|\})$ which reduces the connectivity constraint of Equation (1.3) to

$$
\begin{array}{r}
\forall e \in E \forall v_{x}, v_{y} \in e \exists i, j \in\{1 \ldots|K|\}: \\
\phi\left(v_{x}\right) \in P^{i} \cup P^{j} \wedge \phi\left(v_{y}\right) \in P^{i} \cup P^{j}
\end{array}
$$




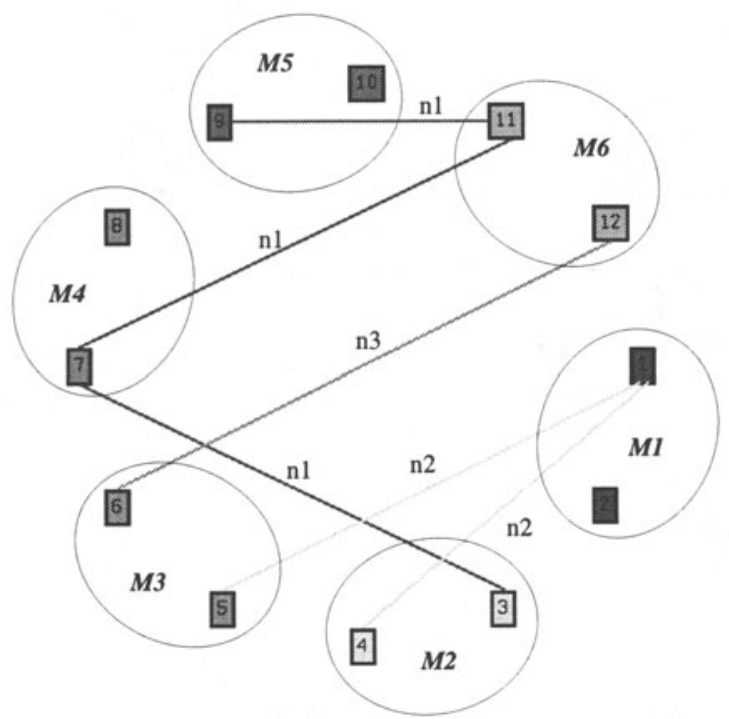

Figure 1.2 Netgraph for which no feasible router configuration exists without splitting.

Our proposed net decomposition strategy consists of two main phases: net selection and net splitting. First a heuristic is applied for finding a suitable net for splitting. Starting with all multi-terminal nets which can be split, i.e. nets attached to modules with free port resources, the set of candidates is ordered by the degree of mobility $M D$. $M D$ is defined as the sum of free ports of all modules $m$ to which net $e$ is attached. We assume that choosing the net with the lowest degree of mobility for splitting will result in the best relaxation of the connectivity constraint for the overall system. After having selected the net to be split we derive a computationally efficient ILP model to determine the splitting module as well as a valid router configuration. The optimality of the ILP approach guarantees that such a router configuration will be found whenever one exists. If this problem turns out to be infeasible, we try to split another net without merging the first one again. In this case we select manually the module $m_{s}$ with the most free port resources as splitting node and reformulate the board-level routing problem. This decomposition strategy is iterated until there are no more free port resources or nets to split, or the board-level routing problem becomes feasible with the relaxed constraints.

Nevertheless, splitting also introduces some cost. Depending on the chosen splitting module $m_{s}$, some additional delay may be incurred on the net as well as additional port resources are allocated in the splitting module itself. Therefore, in the presence of many multi-terminal nets as for example in busdominated communication architectures of embedded systems, the number of 
multi-terminal nets which are split has to be minimized. Otherwise the target system's clock frequency will be reduced unnecessarily or even worse, the limited resources of the execution unit can easily be exceeded. The iterative algorithm proposed above terminates as soon as a feasible solution has been found, rather than splitting all nets as proposed in Mak and Wong, 1995a.

\subsection{ILP FORMULATION}

For finding the splitting node $m_{s}$ as well as a feasible allocation $\phi: V \rightarrow P$ we again use an ILP-formulation. Let $N G(V, E)$ be a netgraph, $E U(M, P, 2)$ an execution unit, $e_{s}$ a net which is to be split, and $M_{s}$ a set of modules at which $e_{s}$ can be split.

The mapping of terminals to ports is again modeled with a set of binary decision variables $X=\left\{x_{i} \mid v_{i} \in V\right\}$ with $x_{i}=0$, if terminal $v_{i}$ is assigned to a port $p \in P^{1}$ and $x_{i}=1$, if $p \in P^{2}$. Additionally, we define a new set of binary decision variables $S=\left\{s_{1}, \ldots, s_{\left|M_{s}\right|}\right\}$ in order to determine the splitting module $m_{s}$. It is $s_{m}=1$, if net $e_{s}$ is split at module $m$, otherwise let $s_{m}=0$. The problem is feasible, if it complies with the following constraints:

- For preserving the connectivity of the target system we require

$$
\forall e \in E \backslash e_{s} \forall v_{i}, v_{j} \in e: x_{i}=x_{j} .
$$

That means all terminals $v_{i}$ of a net are connected via the same router. This is not true for net $e_{s}$ which is available at both routers due to the splitting. Thus, as REPLICA is an execution unit of type $E U(M, K, 2)$ the connectivity constraint given in Equation (1.7) can be eliminated for net $e_{s}$ completely.

- Net $e_{s}$ should be split at exactly one node:

$$
\sum_{m \in M_{s}} s_{m}=1
$$

- For the sake of a more compact notation, we define two new decision variables for the total number of terminals at module $m$ which are assigned to router 1 and router 2 respectively:

$$
\begin{array}{r}
W_{m}^{1}=\sum_{i: \psi\left(v_{i}\right)=m}\left(1-x_{i}\right) \\
W_{m}^{2}=\sum_{i: \psi\left(v_{i}\right)=m} x_{i} .
\end{array}
$$


For all modules which could not serve as splitting module for net $e_{s}$ we only apply the port constraints already introduced in Section 4.1:

$$
\begin{array}{r}
\forall m \in M: \\
W_{m}^{1} \leq\left|P_{m}^{1}\right|=\frac{|P|}{2} \\
W_{m}^{2} \leq\left|P_{m}^{2}\right|=\frac{|P|}{2} .
\end{array}
$$

Let $v_{s}$ be the node at which net $e_{s}$ is split. We have to allocate two ports (one in each port set) for net $e_{s}$ at the splitting module $v_{s}$. Thus, the resulting port constraints are

$$
\begin{array}{r}
\forall m \in M_{s} \exists v_{s} \in e_{s}: \psi\left(v_{s}\right)=m: \\
W_{m}^{1}-\left(1-x_{s}\right)+s_{m} \leq\left|P_{m}^{1}\right| \\
W_{m}^{2}-x_{s}+s_{m} \leq\left|P_{m}^{2}\right|
\end{array}
$$

It should be noted that for all $m \in M_{s}$ with $s_{m}=0$ the port constraints of equations (1.10) and (1.11) are more restrictive than those of equations (1.12) and (1.13) respectively and therefore are effective.

In contrast to the feasibility problem in Section 4.1 particular solutions of this ILP formulation are now rated according to a cost function. Let us define $g_{m}$ as the weight of module $m$. We assign $g_{m}$ with the number of used ports at module $m$ and sum it for all modules net $e_{s}$ is attached to:

$$
\min : \sum_{m \in M_{s}} g_{m} \cdot s_{m}
$$

When minimizing this sum, the module with the most free ports will be chosen as splitting node. Considering the iterative nature of our decomposition strategy this will keep the cardinality of $M_{s}$ for subsequent splitting operations as high as possible. This in turn will relax constraints for $v_{s}$ and improve routability. It should be noted that $g_{m}$ is a constant and can be directly derived from the netgraph.

\section{EXPERIMENTAL RESULTS}

For evaluating the runtime behavior of the ILP formulation of Section $4.1 \mathrm{a}$ benchmark of 21200 different board-level routing problems has been generated. A random generator was used to create different classes of netgraphs $N G(V, E)$ 
with the cardinality $|V|$ as distinguishing feature. Each class consists of 400 different netgraphs. In order to maintain a maximally balanced utilization of the hardware resources the execution unit was also scaled with the cardinality $|V|$. Thus, for all problems an execution unit of type $E U(6,|V|, 2)$ is assumed.

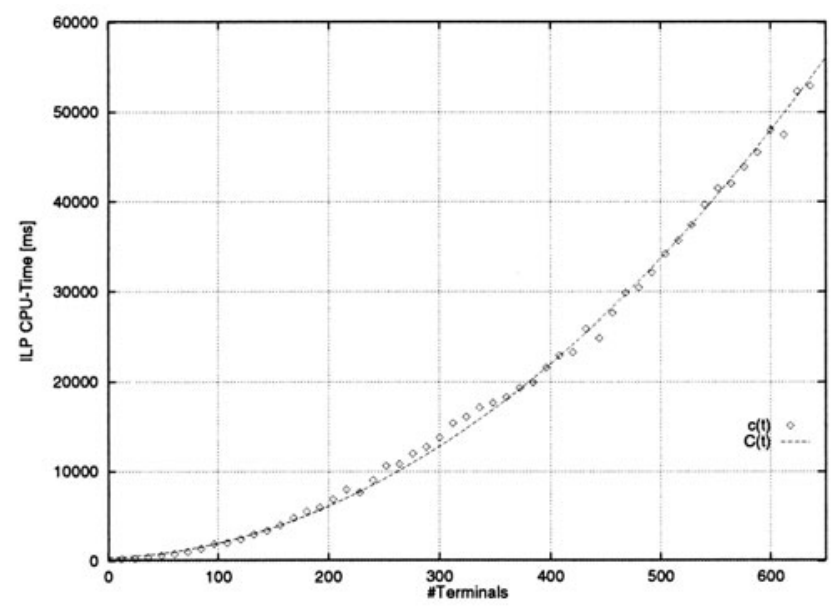

Figure 1.3 Runtime Analysis.

In Figure 1.3 the dependence of the execution time on the cardinality of the investigated netgraph (i.e. the number of terminals) is displayed. The measurements were taken on a Sun UltraSparc (167 MHz, $562 \mathrm{MB} R A M)$ with lp_solve V2.2 Berkelaar, 1997. as ILP-solver. It can be shown that the computational complexity of the particular instance of the BLRP presented in this paper is polynomially bounded. In fact, Figure 1.3 shows that for the benchmark circuits the runtime was bounded by a second degree polynomial.

Figure 1.4 shows the number of infeasible ILP problems in the set of benchmark circuits. It turns out that for execution units of type $E U(6, P, 2)$ almost all problems are feasible for large values of $P$. The investigation of another set of benchmarks with 6041 different netgraphs and an execution unit of type $E U(6,636,2)$ concluded with similar results: only 8 problems $(0.13 \%)$ were infeasible. The decomposition strategy described in Section 5. was applied to the remaining problems, all of which were solved after the first iteration of the algorithm.

\section{CONCLUSIONS}

In this paper we have addressed the board-level routing problem of multiterminal nets in reconfigurable interconnection architectures with coarse granularity. We have presented an ILP-based algorithm which finds a feasible router configuration for a prototyping system consisting of two independent switching 


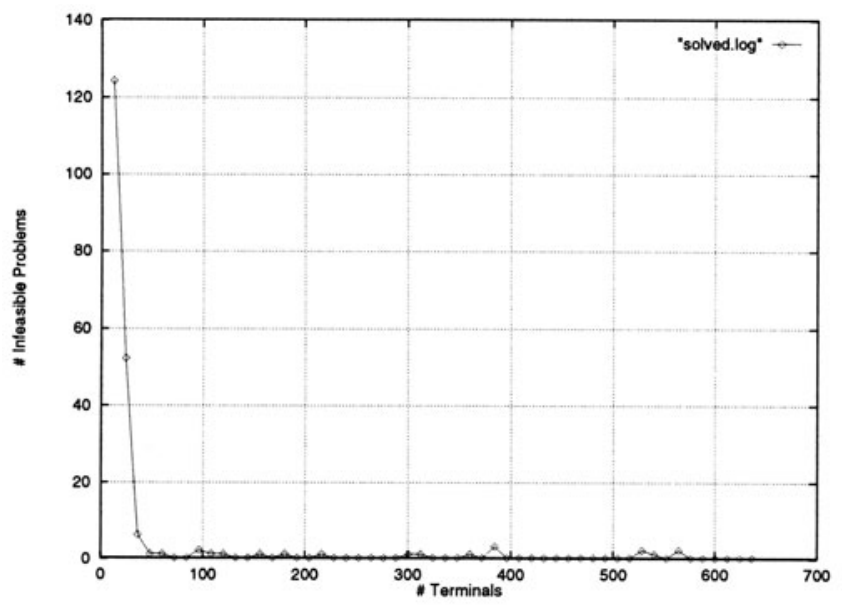

Figure 1.4 Problems which are infeasible without splitting.

devices whenever one exists in polynomial time. Moreover, we have presented an iterative net decomposition strategy for netgraphs which initially cannot be mapped to the execution unit. Instead of decomposing all multi-terminal nets as suggested by previous research, our approach allocates minimal additional port resources to increase routability in the presence of many multi-terminal nets significantly. Given a net to be decomposed, we have also proposed an ILP-based algorithm for finding a splitting node as well as a feasible routing scheme, whenever one exists.

\section{ACKNOWLEDGMENTS}

The work presented here is part of the research program Rapid Prototyping of Embedded Systems with Hard Time Constraints. It is supported by the German Research Foundation Deutsche Forschungsgemeinschaft (DFG) under grant Gl 144/14-1.

\section{Notes}

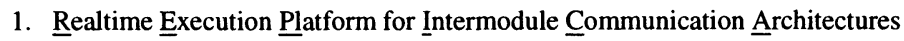

\section{REFERENCES}

(1996). IQX Family Data Sheet. I-Cube Inc.

Berkelaar, M. (1997). lp_solve. Available via anonymous ftp from ftp.es.ele.tue.nl. TU Eindhoven.

Chan, P. and Schlag, M. (1993). Architectural Tradeoffs in Field-ProgrammableDevice-Based Computing Systems. In IEEE Workshop on FPGAs for Custom Computing Machines, pages 138-141. 
Gasteier, M. et al. (1996). An Interactive Approach to Hardware/Software CoDesign. In International Workshop on Logic and Architecture Synthesis, pages 211-218, Grenoble, France.

Gasteier, M. and M., G. (1996). Bus-Based Communication Synthesis on System-Level. In 9th Int. Symposium on System Synthesis, pages 65-70, La Jolla, CA.

Kirschbaum, A., Becker, J., and Glesner, M. (1998). A Reconfigurable HardwareMonitor for Communication Analysis in Distributed Real-Time Systems. In 5th Reconfigurable Architectures Workshop RAW98, Orlando, USA.

Kirschbaum, A. and Glesner, M. (1997). Rapid Prototyping of Communication Architectures. In IEEE Workshop on Rapid System Prototyping, pages 136141, Chapel Hill, USA.

Lawler, E. (1976). Combinational Optimization: Networks and Matroids. Holt, Rinehart and Winston, New York.

Mak, W. and Wong, D. (1995a). Board-Level Multi-Terminal Net Routing for FPGA-based Logic Emulation. In IEEE/ACM Int. Conference on Computer Aided Design, pages 339-344.

Mak, W. and Wong, D. (1995b). On Optimal Board-Level Routing for FPGAbased Logic Emulation. In ACM/IEEE Proc. 32nd Design Automation Conference, pages 552-556.

Ortega, R. and Borriello, G. (1997). Communication Synthesis for Embedded Systems with Global Considerations. In Fifth Int. Workshop on Hardware/Software Codesign, pages 69-73.

Slimane-Kadi, M., Brasen, D., and Saucier, G. (1994). A Fast-FPGA Prototyping System that uses inexpensive High-performance FPIC. In ACM/SIGDA Int. Workshop on Field-Programmable Gate Arrays.

Varghese, J. et al. (1993). An Efficient Logic Emulation System. IEEE Transactions on VLSI, 1(2):171-174.

Yen, T.-Y. and Wolf, W. (1995). Communication Synthesis for Distributed Embedded Systems. In International Conference on Computer Aided Design, pages 288-294. IEEE Computer Society Press. 